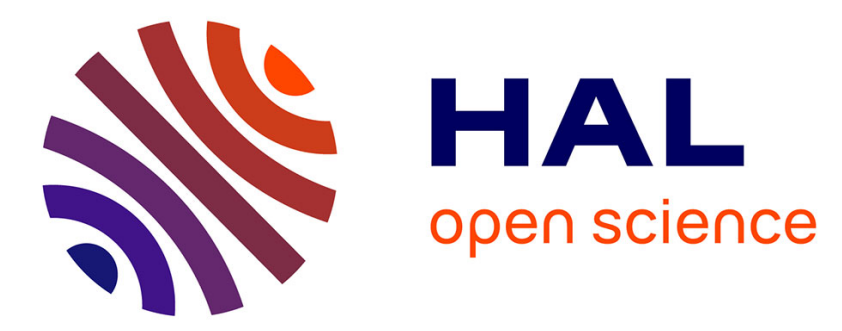

\title{
Impaired Sense of Agency and Associated Confidence in Psychosis
}

Amit Regev Krugwasser, Yoni Stern, Nathan Faivre, Eiran Vadim Harel, Roy Salomon

\section{> To cite this version:}

Amit Regev Krugwasser, Yoni Stern, Nathan Faivre, Eiran Vadim Harel, Roy Salomon. Impaired Sense of Agency and Associated Confidence in Psychosis. npj Schizophrenia, 2022, 10.1038/s41537022-00212-4 . hal-03318445

\section{HAL Id: hal-03318445 \\ https://hal.science/hal-03318445}

Submitted on 10 Aug 2021

HAL is a multi-disciplinary open access archive for the deposit and dissemination of scientific research documents, whether they are published or not. The documents may come from teaching and research institutions in France or abroad, or from public or private research centers.
L'archive ouverte pluridisciplinaire HAL, est destinée au dépôt et à la diffusion de documents scientifiques de niveau recherche, publiés ou non, émanant des établissements d'enseignement et de recherche français ou étrangers, des laboratoires publics ou privés. 
Impaired Sense of Agency and Associated Confidence in Psychosis

\section{Running title: Aberrant Agency and Metacognition in Psychosis}

Amit Regev Krugwasser ${ }^{1}$, Yonatan Stern ${ }^{1,2}$, Nathan Faivre ${ }^{3}$, Eiran Vadim Harel ${ }^{4,5}$, Roy Salomon $^{1}$

${ }^{1}$ Gonda Brain Research Center, Bar-Ilan University, Ramat Gan, Israel

${ }^{2}$ Psychology Department, University of Haifa, Haifa, Israel

${ }^{3}$ Univ. Grenoble Alpes, Univ. Savoie Mont Blanc, CNRS, LPNC, Grenoble, France

${ }^{4}$ Beer Yaakov-Ness Ziona Mental Health Center, Beer Yaakov, Israel

${ }^{5}$ Sackler Faculty of Medicine, Tel Aviv University, Tel Aviv, Israel

Correspondence

Name: Amit Regev Krugwasser

Email: amitregev3@gmail.com

Address: The Leslie and Susan Gonda (Goldschmied) Multidisciplinary Brain Research Center, Bar- Ilan University, Building number 901, Ramat- Gan 5290002, Israel

Phone number: +97235317755

Fax number: +97235352184 


\section{Abstract}

The Sense of Agency (SoA), our sensation of control over our actions, is a fundamental mechanism for delineating the Self from the environment and others. SoA arises from implicit processing of sensorimotor signals as well as explicit higher-level judgments. Psychosis patients suffer from difficulties in the sense of control over their actions and accurate demarcation of the Self. Moreover, it is unclear if they have metacognitive insight into their aberrant abilities. In this pre-registered study, we examined SoA and its associated confidence judgments using an embodied virtual reality paradigm in psychosis patients and controls. Our results show that psychosis patients not only have a severely reduced ability for discriminating their actions but they also do not show proper metacognitive insight into this deficit. Furthermore, an exploratory analysis revealed that the SoA capacities allow for high levels of accuracy in clinical classification of psychosis. These results indicate that SoA and its metacognition are core aspects of the psychotic state and provide possible venues for understanding the underlying mechanisms of psychosis, that may be leveraged for novel clinical purposes.

Keywords: Sense of Agency, Metacognition, Psychosis, Virtual Reality, Clinical Classification 


\section{Introduction}

Psychosis is a severe psychiatric condition which includes numerous symptoms in which the delineation of the Self is compromised. Psychosis patients often report sensations of loss of control over their thoughts or actions, which has led to the suggestion that deficits in the demarcation of the Self constitute a core aspect of psychosis and schizophrenia spectrum disorders (Hur et al., 2014; Raballo et al., 2011; Sass \& Parnas, 2003; Schneider, 1959). A central process giving rise to the sense of Self is the Sense of Agency (SoA), the feeling of control over one's actions. Research has highlighted the role of SoA in delineating one's bodily and mental functions from the environment and conspecifics, allowing one's experience as a distinct embodied agent in the world (Gallagher, 2012, 2000; Haggard, 2017; Krugwasser et al., 2019). Contemporary theories suggest that SoA is based on pre-reflexive predictive sensorimotor processes (Bays et al., 2006; David et al., 2008; Haggard, 2017; Wolpert et al., 1995), as well as explicit processes that take into account contextual and conceptual factors (Moore et al., 2009; Synofzik et al., 2008). Within this theoretical framework, actions are accompanied by efferent copies that generate predictions regarding the expected sensory outcomes of these actions. Incoming afferent sensory information is then compared to the predictions. If the two match, the action is ascribed to the Self and accompanied by a SoA. These predictive mechanisms allow one to suppress the consequences of one's actions both at the perceptual (Bays et al., 2006; Blakemore et al., 1998; Kilteni \& Ehrsson, 2017) and the neural level (Hughes \& Waszak, 2011; Palmer et al., 2016; Shergill et al., 2012; Stripeikyte et al., 2020; Van Elk et al., 2014). However, if a mismatch occurs the sensory outcomes are ascribed to an 
external origin and are passed up the hierarchy to explicit processes that explain them in light of beliefs, knowledge and other contextual factors. Thus the integration of efferent predictive models and afferent sensory signals shape SoA and play a key role in delineating the Self (Hughes et al., 2013; Synofzik et al., 2009).

Disturbances of SoA are a striking aspect of psychosis, common across schizophrenia spectrum disorders (Franck et al., 2001; Frith \& Done, 1989; Haggard et al., 2003; Hauser et al., 2011; Maeda et al., 2012; Voss et al., 2010). It has been suggested that aberrant hierarchical prediction mechanisms underlie psychosis symptoms (Corlett et al., 2019; Fletcher \& Frith, 2008; Sterzer et al., 2018) and specifically abnormal SoA (Frith \& Done, 1989; Leptourgos \& Corlett, 2020). Accordingly, psychosis patients exhibit reduced sensory and neural attenuation for actions (Ford et al., 2013; Shergill et al., 2005, 2014), impaired ability to predict the outcomes of their actions (Lindner et al., 2005; Synofzik et al., 2010; Voss et al., 2010) and erroneous explicit judgments of agency (Fourneret et al., 2002; Franck et al., 2001). Thus, abnormal sensorimotor predictive mechanisms may induce inaccurate experiences of agency, causing confusion between self and externally induced sensations. Indeed, recent research has demonstrated a causal relationship between predictive processes and demarcation of the Self. For example, inducing tactile sensorimotor conflicts caused auditory selfdiscrimination deficits in first episode psychosis patients (Salomon et al., 2020), and psychosis-like symptoms in healthy participants (Bernasconi et al., 2021; Blanke et al., 2014; Faivre, Vuillaume, et al., 2020; Serino et al., 2020).

However, most studies of SoA have employed non embodied paradigms in which action-outcomes contingencies are acquired during the experiment (e.g., press a button 
- hear a tone). While these paradigms have enriched our understanding of learned action outcome mechanisms, they do not tap into the strong predictive capacities afforded by a lifelong experience of controlling our bodies (Stern et al., 2020). Thus, embodied SoA may differ in regards to the strength of the priors of the predictive processes (Allen \& Tsakiris, 2018; Leptourgos \& Corlett, 2020), and better capture psychotic patients' anomalous self-experiences (Sass \& Parnas, 2003).

While deficits in SoA have been found across the schizophrenia spectrum, it is yet unclear whether patients are aware of this impairment. Metacognitive deficits, involving lack of insight into their condition, are commonly found in psychosis and are associated with poorer prognosis (Koren et al., 2006; Lysaker et al., 2005; Lysaker \& Dimaggio, 2014). However, recent research has shown that metacognitive capacities for some simple perceptual tasks do not seem to be deficient in schizophrenia patients (Faivre, Roger, et al., 2020; Rouy et al., 2021). Awareness of control over our actions, is critical for meaningful interactions with the world. While there have been some suggestions that SoA itself is a metacognitive mechanism (Chambon et al., 2014, but see Constant et al., 2021), to date there has been no study of metacognitive abilities of embodied SoA in psychosis.

The current pre-registered study examined embodied SoA and metacognition in psychosis patients and healthy participants. We employed a virtual hand (VH) paradigm previously used in healthy participants (Krugwasser et al., 2019; Stern et al., 2020) in which we manipulate the sensorimotor correspondence between the participants' real hand movement and the displayed VH's movement by inserting a temporal or spatial alteration. First, we hypothesized that patients' embodied SoA, operationalized as their 
ability to detect sensorimotor conflicts, would be impaired for both temporal and spatial alterations. Second, we hypothesized that their metacognition of SoA, operationalized as the correspondence between accurate sensorimotor conflict detection and associated confidence ratings, would be diminished compared to healthy participants. Finally, in an exploratory analysis we examined whether we could accurately classify psychosis and control participants based on task performance using an automated classifier, thereby probing the task's clinical utility. Pre-registration is available at https://bit.ly/2US57bX, code and data are available at github.com/amitrekru/SoA Metacognition Psychosis.

\section{Methods}

\section{Participants}

Healthy controls $(H C)$. Thirty-four control participants that self-reported no psychiatric or neurological history from Bar-llan University participated in the experiment. Four participants were excluded from the analysis (see pre-registration and supplementary material section A for criteria and details) leaving a total of 30 healthy participants (mean age: 24.4 years, STD: 3 years, 15 females).

Psychosis patients. Thirty-one participants with psychosis from Beer Yaakov-Ness Ziona Mental Health Center participated in the experiment. One participant was excluded from the analysis (see pre-registration and supplementary material section $A$ for criteria and details) leaving a total of 30 psychosis participants (mean age: 32 years, STD: 9.2 years, all males. See Table 1 for clinical characteristics). Patients at the time 
of the experiment were hospitalized and under pharmacological treatment (see supplemental Table S1 for medication details).

All participants gave written informed consent, were right-handed, with normal or corrected-to-normal vision and naïve to the purpose of the experiment. The experiment was performed in accordance with the ethical standards of the Declaration of Helsinki and the experimental protocols were approved by the Gonda Multidisciplinary Brain Research Center ethics committee (for $\mathrm{HC}$ ) and by the Beer Yaakov-Ness Ziona Mental Health Center ethics committee (for psychosis participants).

\begin{tabular}{|c|l|l|l|l|l|}
\hline \multirow{2}{*}{ Group } & \multicolumn{1}{|c|}{ Diagnosis (N) } & \multicolumn{1}{|c|}{ Age } & \multicolumn{1}{c|}{$\begin{array}{c}\text { PANSS } \\
\text { Positive }\end{array}$} & $\begin{array}{c}\text { PANSS } \\
\text { Negative }\end{array}$ & \multicolumn{1}{c|}{$\begin{array}{c}\text { PANSS } \\
\text { Total }\end{array}$} \\
\hline \multirow{5}{*}{ Psychosis } & Schizophrenia (17) & $30.6(7.2)$ & $14.6(3.1)$ & $16.6(4.7)$ & $64.3(12.4)$ \\
\cline { 2 - 6 } & Schizoaffective disorder (5) & $35(13.5)$ & $15.2(2.6)$ & $9.4(2.4)$ & $52.8(7.5)$ \\
\cline { 2 - 6 } & Active psychosis (4) & $29.3(8.8)$ & $17.3(4.3)$ & $16.5(6.8)$ & $67.5(13.4)$ \\
\cline { 2 - 6 } & Paranoid schizophrenia (2) & $28.5(2.1)$ & $12(1.4)$ & $16(4.2)$ & $59(9.9)$ \\
\cline { 2 - 6 } & Bipolar Disorder (2) & $28(8.5)$ & $18(0)$ & $13.5(6.3)$ & $69.5(19)$ \\
\cline { 2 - 6 } & Mean & $\mathbf{3 0 . 9 ( 8 . 3 )}$ & $\mathbf{1 5 . 1 ( 3 . 2 )}$ & $\mathbf{1 5 . 2 ( 5 . 2 )}$ & $\mathbf{6 2 . 8 ( 1 2 . 4 )}$ \\
\hline \multirow{2}{*}{ Control } & None (30) & $24.4(3)$ & & & - \\
\hline
\end{tabular}

Table 1. Participants' demographic and clinical characteristics. PANSS = Positive and Negative Syndrome Scale. Numbers represent the mean, numbers within the parenthesis represent the standard deviation.

Experimental procedure. Participants' right hand was occluded from their view and placed below a Leap Motion controller (Leap Motion Inc., San Francisco, CA) that tracked their hand's movement. A realistic 3D VH that mimicked the real hand's movement was displayed on a monitor (see supplementary material section A and Krugwasser et al., 2019 for further details). Each trial began with a fixation cross, 
followed by presentation of the $\mathrm{VH}$ during which participants performed a single bending movement with their index finger. In $25 \%$ of the trials, the VH's movement was identical to the real hand's movement, while in $75 \%$ of the trials a sensorimotor alteration was introduced. Three magnitudes of sensorimotor alterations were presented in temporal or spatial aspects. In the temporal aspect, the VH's movement was delayed (100/200/300 ms; Krugwasser et al., 2019; Salomon et al., 2013; Shimada et al., 2009; Wen et al., 2015), and in the spatial aspect an angular deviation of the VH's index finger's was inserted (i.e., its lateral trajectory was diverged towards the thumb by $6 / 10 / 14^{\circ}$; Franck et al., 2001; Kannape et al., 2010; Krugwasser et al., 2019). Importantly, only a single alteration (or none) was presented in each trial. Each magnitude of alteration per aspect was presented 30 times, in a random order across five blocks, resulting in a total of 240 trials. Following the VH presentation, participants responded to a Yes/No question "Was the movement of the $\mathrm{VH}$ identical to my movement?", measuring SoA via the perceived congruence between the action and its outcome (Franck et al., 2001; Krugwasser et al., 2019; Stern et al., 2020). Participants then rated their confidence in the agency judgment on a continuous slider ranging from 'Not confident' (i.e., -3) to 'Very confident' (i.e., 3; see Fig. 1 for paradigm flow chart). Finally, the clinical symptoms of the psychosis patients were assessed using the Positive and Negative Syndrome Scale (PANSS, see Kay et al., 1987), and HC participants completed the Schizotypal Personality Questionnaire-Brief Version (SPQ-B, see Raine \& Benishay, 1995). 
(i)

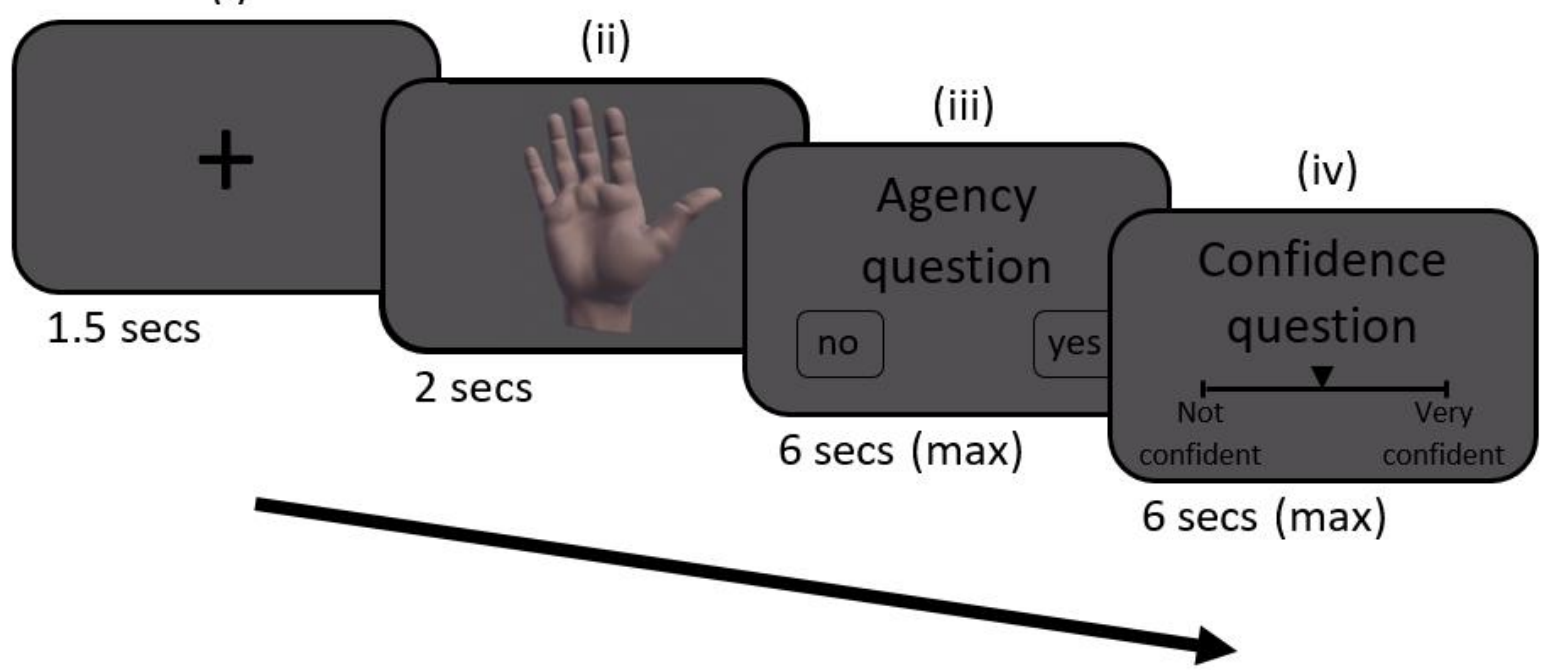

Figure 1. Trial flow. Each trial began with a fixation cross (i), followed by the VH presentation (ii), agency question (iii) and the confidence question (iv).

Data analysis. Data was pre-processed using in-house Matlab scripts (MATLAB, 2019). Following the pre-registration, trials in which no movement was made, camera malfunctioned, or participants failed to respond were removed from subsequent analyses (1.9\% and $8.4 \%$ of the trials for $\mathrm{HC}$ and psychosis patients respectively). Statistical analyses and visualization were performed in R (R Core Team, 2019).

SoA. SoA (i.e., self-attributing the VH's movement) was analyzed by comparing a series of logistic mixed-effects regressions implemented in the 'Ime4' package (Bates et al., 2014). Following Barr et al., (2013), we attempted to include maximal random effects that also allow for model convergence. Models were compared using the differences of their Bayesian Information Criteria (i.e., $\Delta$ BIC, see Schwarz, 1978), with values between 2 and 6 , between 6 and 10 and $>10$ considered as positive, strong and very strong evidence respectively for the model with the lower value (Kass \& Raftery, 1995; 
Wagenmakers, 2007). The winning model's fixed parameters' significance were derived using the Satterthwaite's degrees of freedom approximation and type III error implemented in the 'ImerTest' package (Kuznetsova et al., 2017). Signal detection measures of sensitivity and bias ( $d^{\prime}$ and $c$, respectively; See Macmillan, 2002) of SoA were also calculated, across magnitudes of alteration.

Confidence ratings. Similar to SoA, confidence was analyzed by comparing a series of linear mixed-effects regression models. Following an observed hyperbolic effect of Alteration Magnitude on Confidence (see Fig. 2B and supplemental Fig. 2S), a quadratic expansion of Alteration Magnitude was used as a fixed parameter. Metacognitive performance was also assessed using the gamma $(\mathrm{Y})$ ranked correlations (Goodman \& Kruskal, 1979) between confidence ratings and accuracy. Gamma correlations range from minus one to one, with a value of zero indicating that there is no association between accuracy and confidence. Gamma was calculated across the magnitudes of alteration for each participant.

Correlations with clinical measures. In line with our pre-registration, Pearson correlations were used to examine the relation between sensitivity and clinical ratings (i.e., PANSS scores for psychosis patients, and SPQ-B scores for HC). To further inspect the relation between performance and clinical measures, this analysis was supplemented with an exploratory analysis of the correlations between criterion, metacognitive performance and clinical ratings, as well as metacognitive performance and clinical ratings. 
Clinical classification based on SoA performance. In an exploratory analysis we examined the potential clinical utility of our SoA paradigm for classification of participants to psychosis or control groups based on their SoA performance. We developed an algorithm that classifies a given participant based on the comparison of his/her SoA judgments' linear fit's slope, to the mean slopes of both groups. Participants were classified to the group with the smaller Euclidean distance (from each group linear fit's slope) combined across aspects of alteration (see Fig. 3A). The algorithm repeats this process 10,000 times, randomly leaving out the same proportion of participants from each group. Furthermore, we used the classifier with different proportions of leftout-participants as well as smaller subsets of trials for each participant (see Fig. 3C, left panel).

\section{Results}

Impaired SoA in psychosis patients. In line with our pre-registered hypothesis, the best model included the main effects of Alteration Magnitude, Group and their interaction. This model was better $(\Delta \mathrm{BIC}=3.9)$ than the next model that included the same terms in addition to Aspect and its interactions. The intercept and slope of Alteration Magnitude were included as random effects (see supplementary material section $\mathrm{C}$ for full details of models). There was a significant main effect of Alteration Magnitude $(\beta=-1.18, p<0.0001, Z=18.2,95 \% \mathrm{Cl}[-1.31,-1.05])$, such that as magnitude increased SoA ratings decreased across groups. There was a significant main effect of Group ( $\beta=-0.61, p<0.0001, Z=5.9,95 \% \mathrm{Cl}[-0.82,-0.41])$, with the psychosis group showing an increased tendency to self-attribute the observed movements across the magnitudes of alteration. Notably, as predicted, there was a 
significant interaction between Alteration Magnitude and Group $(\beta=-0.52, p<0.0001, Z$ $=8.1,95 \% \mathrm{Cl}[-0.65,-0.4])$, resulting from the psychosis group's moderate decrease in SoA ratings as alteration magnitude increased in comparison to HC's steep decrease in SoA as magnitude increased. Importantly, similar results were obtained for different random effects structure (see supplementary material section C). Complementing our finding of impaired SoA using mixed models, an independent samples t-test of sensitivity and bias revealed that participants in the control group had higher sensitivity and lower bias than the psychosis patients $\left(d_{\text {Control }}^{\prime}=1.8, d_{\text {Psychosis }}^{\prime}=0.76, t_{56}=7.39\right.$, Cohen's $d=1.9, p<0.0001 ; C_{\text {Control }}=-0.43, C_{\text {Psychosis }}=-0.73, t_{52}=2.88$, Cohen's $d=$ $0.74, p<0.01$, see supplemental Fig. S1). Thus, in line with our pre-registration, the psychosis group exhibited impaired SoA.

Impaired metacognition in psychosis patients. In line with our preregistered hypothesis, the best model included all main effects and interactions of Alteration Magnitude, Group and SoA Accuracy (i.e., was the SoA judgment correct), with very strong evidence $(\triangle \mathrm{BIC}=70)$ over a model that did not include Group and its interactions. The intercept and slope of Alteration Magnitude were included as random effects. Examining the winning model's parameters, we found a significant three-way interaction between Alteration Magnitude, Group and Accuracy $(\beta=0.18, p<.001, t=$ $6.1,95 \% \mathrm{Cl}[0.12,0.24])$. This interaction was driven by the psychosis group's consistently higher confidence ratings despite their low levels of accuracy especially in trials with large alteration magnitudes (see Fig. 2B and supplemental Fig. 2S). Thus, the psychosis group exhibited impaired metacognitive capacities as their confidence ratings did not track their accuracy in comparison to the HCs. In addition, there was a main 
effect of Alteration Magnitude $(\beta=-0.18, p<.001, t=7.47,95 \% \mathrm{Cl}[-0.13,-0.23])$, reflecting the increased confidence when there was either an extreme alteration or none. Likewise, a main effect of Accuracy was found ( $\beta=-0.47, p<.001, t=11.44$, $95 \% \mathrm{Cl}[-0.55,-0.39])$, reflecting that across groups, confidence was increased when SoA judgments were correct. In contrast, Group was not significant $(\beta=-0.18, p=0.07$, $t=1.78,95 \% \mathrm{Cl}[-0.39,0.02])$, thus overall confidence ratings between groups were not significantly different. Importantly, similar results were obtained for different random effects structure (see supplementary material section C).

Further comparing the groups' metacognitive performance using gamma ranked correlation between confidence and accuracy, we found that the $\mathrm{HC}$ group exhibited a higher correlation $(y=0.26,95 \% \mathrm{Cl}[0.17,0.35])$ in comparison to the psychosis group $(y=-0.02,95 \% \mathrm{Cl}[-0.12,0.08]$; see Fig. $2 \mathrm{C})$, and this difference was significant $\left(t_{57}=\right.$ 4.27, $p<0.0001$, Cohen's $d=1.1$ ). Examining whether each group's gamma significantly differed from zero (i.e., no correlation between accuracy and confidence) via a one-sample t-test, $\mathrm{HC}$ 's gamma distribution was significantly higher than zero $\left(t_{29}=\right.$ $5.8, p<0.001$, Cohen's $d=1.07$ ), whereas the psychosis group's was not significantly different from zero $\left(t_{29}=0.49, p=0.63\right.$, Cohen's $\left.d=0.09\right)$. These findings complement the three-way interaction found in the mixed-models, demonstrating that psychosis patients exhibit impaired metacognition and their confidence ratings do not track their accuracy. 
$\underline{\text { Temporal }}$

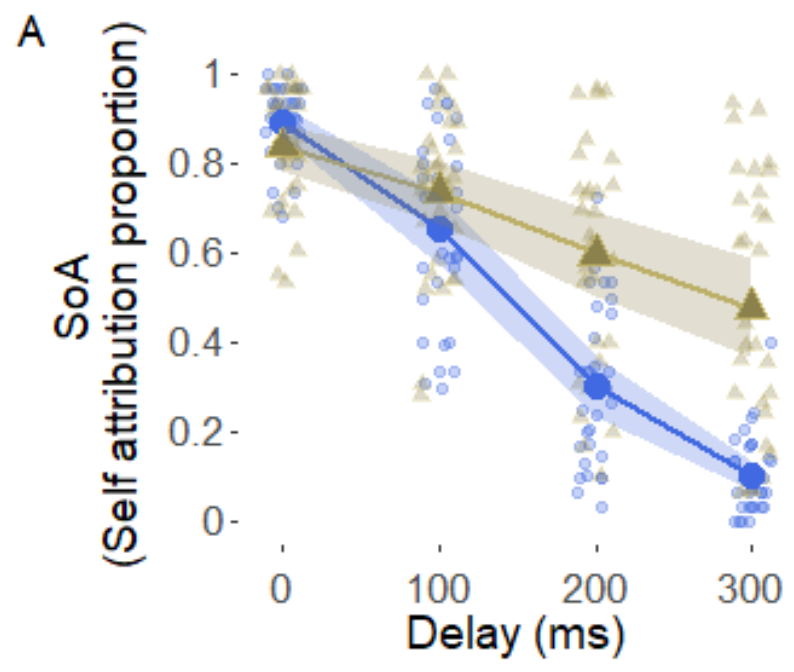

B

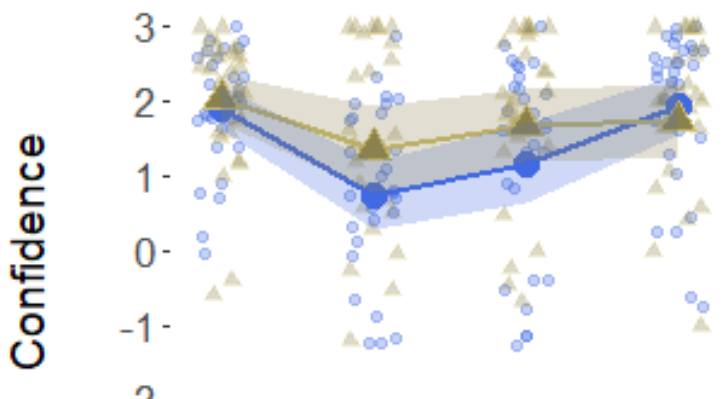

-2 -

$-3-$

$\begin{array}{llr}0 & 100 \quad 200 & 300 \\ & \text { Delay }(\mathrm{ms})\end{array}$

C

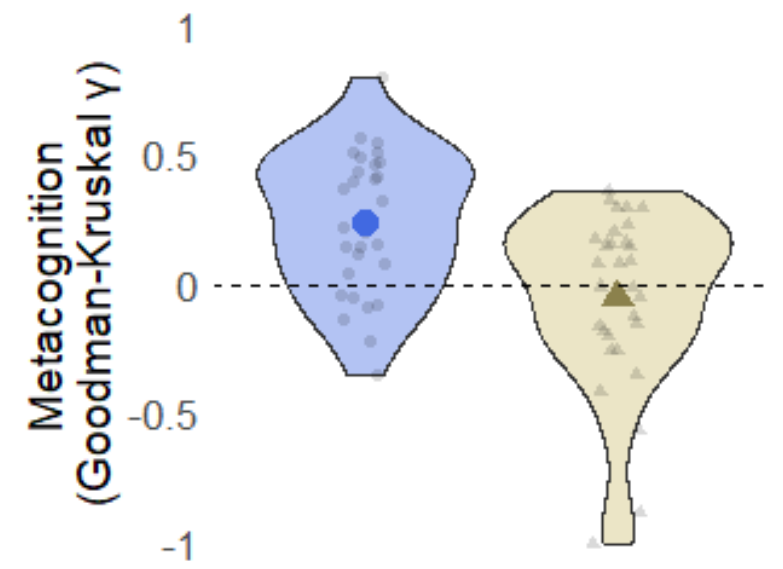

Spatial
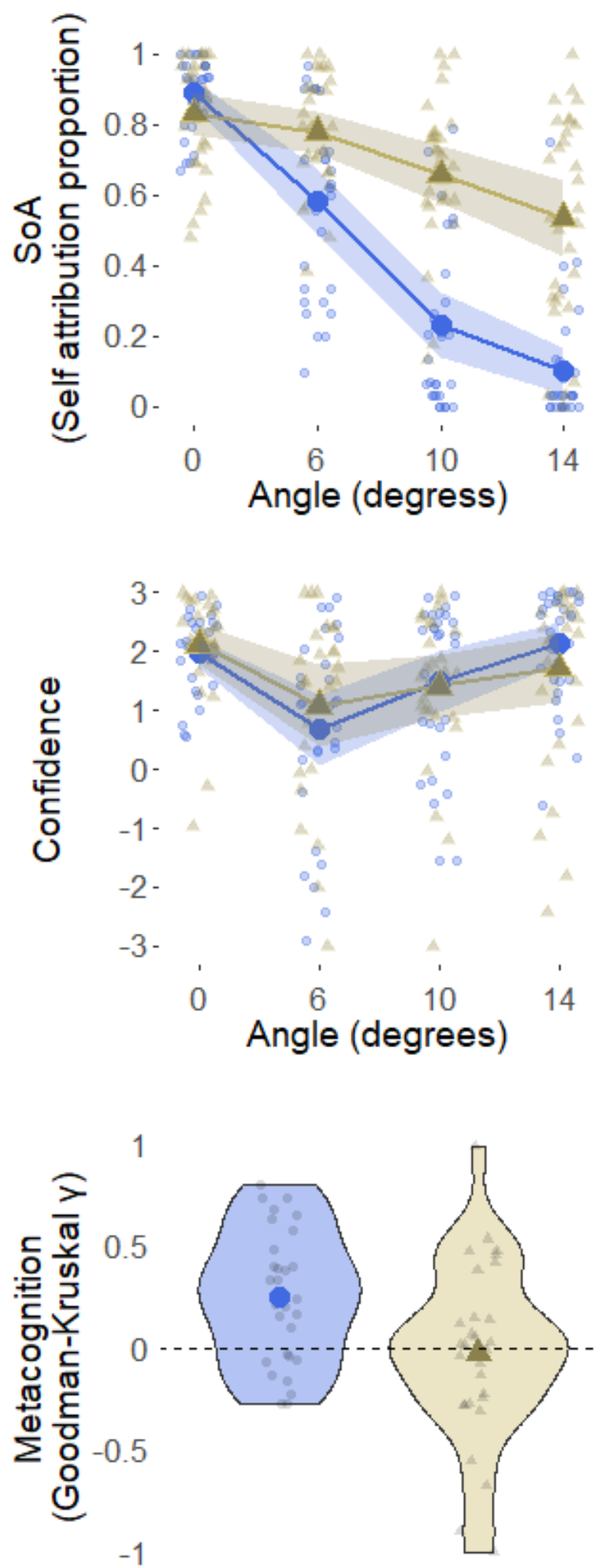

Group - HC $\perp$ PSY 
Figure 2. Group mean and individual ratings of SoA, confidence and metacognitive performance. (A) Self attribution in the temporal aspect (left) and in the spatial aspect (right). Shaded area represents $95 \% \mathrm{Cl}$, large shapes represent group means. (B) Confidence in the temporal (left) and spatial (right) aspects following correct answers to the SoA question. (C) Distribution of metacognitive performance in the temporal (left) and spatial (right) aspects.

Correlation between task performance and clinical measures. In psychosis patients, contrary to our hypothesis, we did not find a significant correlation between sensitivity and the total PANSS score $(r=-0.03, p=0.86)$ nor its subscales (see Table 2). Likewise, bias was not significantly correlated with the total PANSS score ( $r=0.04, p$ $=0.84$ ) nor its subscales (see Table 2). In an exploratory analysis, we found that metacognitive performance was significantly negatively correlated with the PANSS Positive subscale score ( $r=-0.47, p<0.01$, uncorrected for multiple comparisons), such that metacognitive performance was higher in patients with fewer positive symptoms.

In HC, contrary to our hypothesis, we did not find a significant correlation between sensitivity and schizotypy (i.e., total SPQ-B score) $(r=-0.12 p=0.53)$ nor its subscales (see Table 2). Likewise, bias was not significantly correlated with the total SPQ-B score $(r=0.32, p=0.08)$, yet it was significantly correlated with SPQ-B Disorganization subscale ( $r=0.48, p<0.001$, uncorrected for multiple comparisons). 


\begin{tabular}{|c|l|c|l|l|}
\hline Group & \multicolumn{1}{|c|}{ Scale } & \multicolumn{1}{|c|}{$\mathbf{d}^{\prime}$} & \multicolumn{1}{|c|}{$\mathbf{c}$} & $\begin{array}{l}\text { Goodman- } \\
\text { Kruskal Y }\end{array}$ \\
\hline \multirow{4}{*}{ Control } & SPQ-B Cognitive-perceptual deficits & -0.32 & 0.24 & 0.21 \\
\cline { 2 - 5 } & SPQ-B Interpersonal deficits & 0.23 & 0.02 & 0.2 \\
\cline { 2 - 5 } & SPQ-B Disorganization & -0.25 & $0.48^{* *}$ & 0.11 \\
\cline { 2 - 5 } & SPQ-B Total & -0.12 & 0.32 & 0.26 \\
\hline \multirow{3}{*}{ Psychosis } & PANSS Positive & -0.16 & 0.01 & $-0.47^{* *}$ \\
\cline { 2 - 5 } & PANSS Negative & 0.11 & 0.04 & -0.17 \\
\cline { 2 - 5 } & PANSS General & -0.08 & 0.05 & -0.08 \\
\cline { 2 - 6 } & PANSS Total & -0.03 & 0.04 & -0.23 \\
\hline
\end{tabular}

Table 2. Correlation between SoA performance and clinical measures. Correlations between clinical measures and sensitivity, bias \& metacognition. ${ }^{* *} p<0.01$ (uncorrected for multiple comparisons).

Group classifier. Overall, the classifier was able to accurately classify participants in $89 \%$ of the cases (see Fig. 3B). Using a two-sample Kolmogorov-Smirnov test for a difference between distributions, we found this accuracy rate to be significantly higher than chance level ( $D=0.95, p<0.001$, tested by randomly labeling participants as control or patients, and comparing the accuracy rate of the classifier to the actual accuracy rate; See supplemental Fig. S3). This finding was robust across different proportions of trials and participants left out, such that using only half the trials (i.e., 120 trials) and leaving out $80 \%$ of the participants (i.e., 24 out of 30 per group), only decreased the classifier performance to $85 \%$ accuracy. To further examine the realworld applicability of our task, we also examined classification by sampling trials from the first block of the experiment only. This excludes the possibility that the high classification accuracy rates are dependent on the participants' learning along the task. Using only the first 48 trials and leaving out $20 \%$ of the participants, we obtained $81 \%$ 
accuracy, that was reduced to $73 \%$ when using only 24 trials and leaving out $80 \%$ of the participants (see Fig. 3C, right panel). 
A

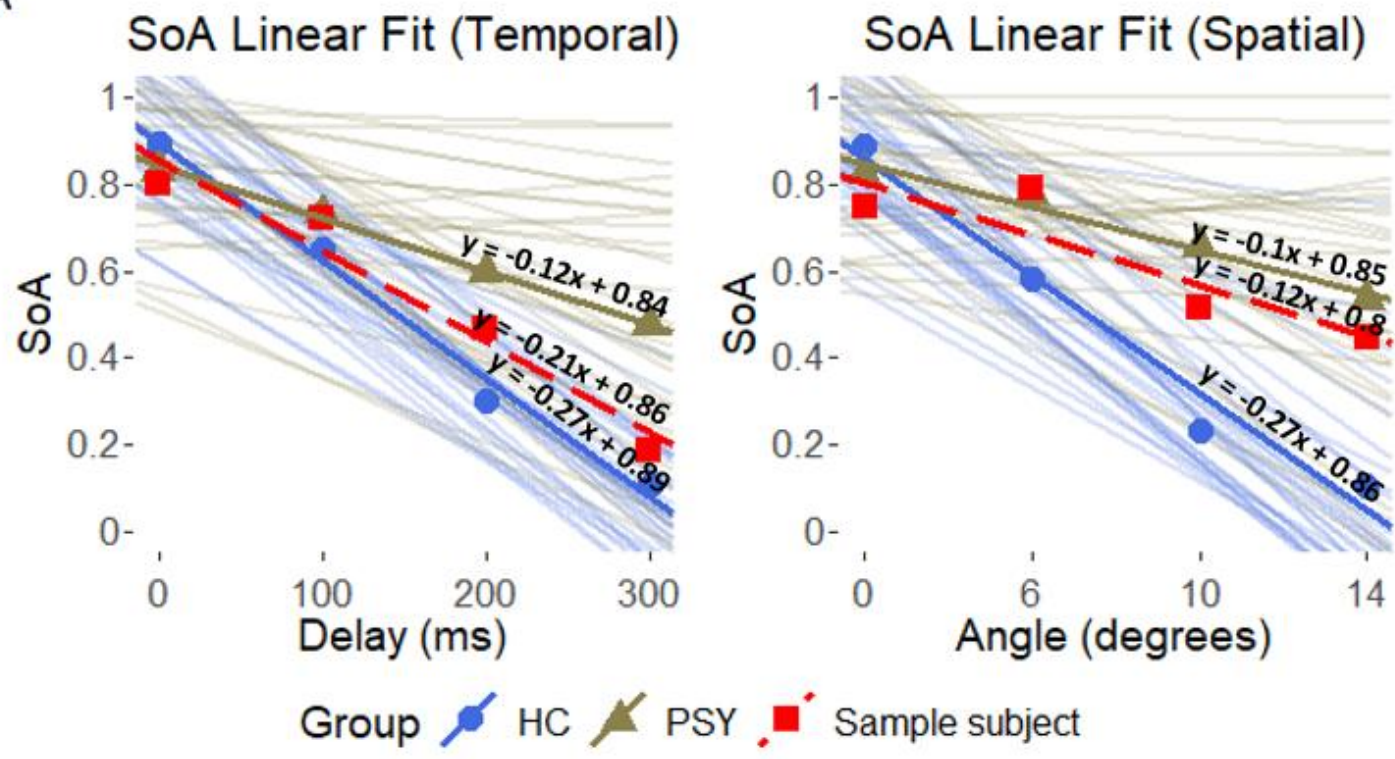

B

Accuracy -

Sensitivity -

Specificity -

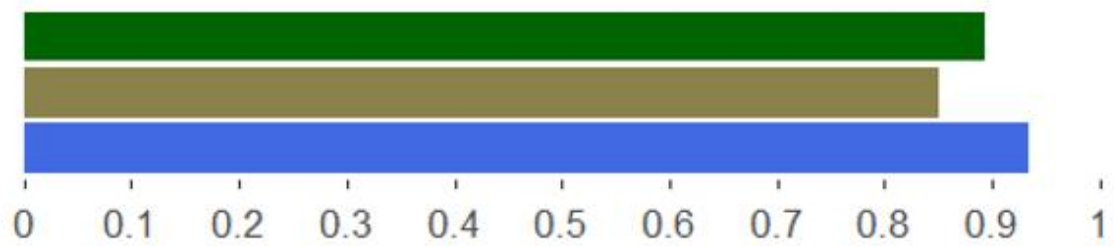

C

Entire Experiment

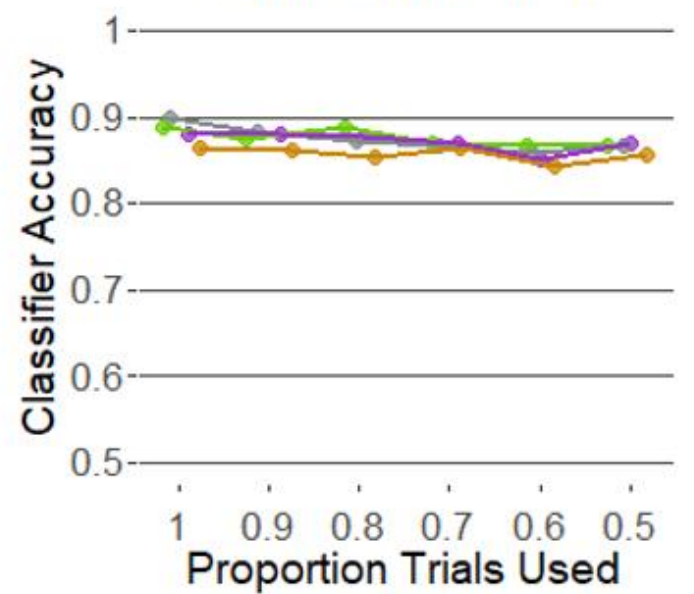

First Block

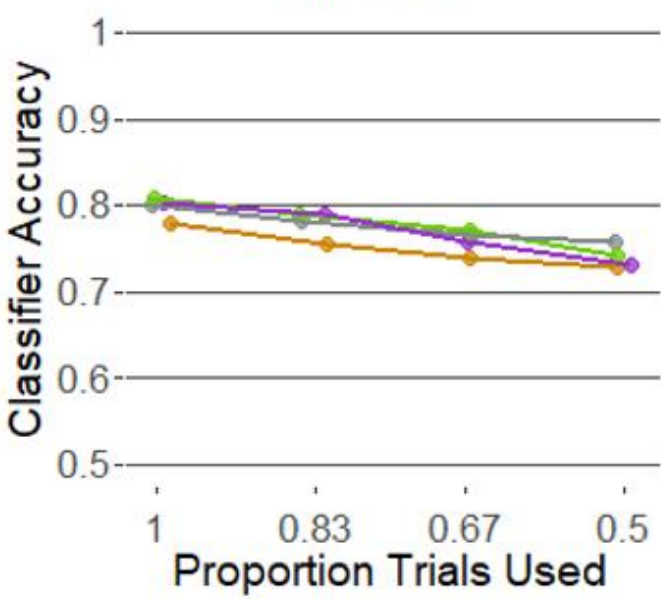

$\%$ Participants Left Out $\rightarrow 20 \multimap 40 \multimap 60 \multimap 80$ 
Figure 3. Group classifier performance. (A) Classification of two sample subjects (exemplar control participant and psychosis patient, linear fit in dashed red line), that are accurately classified as "control" (left) and "psychosis" (right). (B) Classifier performance, leaving out $20 \%$ of the participants (i.e., 6) in each iteration. Sensitivity is the percent of psychosis patients correctly classified, specificity is the percent of $\mathrm{HC}$ correctly classified. (C) Classifier accuracy across different proportions of participants left out and number of trials sampled. Trials were randomly sampled from the entire experiment (left panel), or from the first block (right panel).

\section{Discussion}

Employing an ecological VR paradigm, we examined embodied SoA and associated confidence ratings in healthy and psychosis patient populations. Our results revealed several important findings. First, psychosis participants showed an extensive deficit in SoA, and were impaired in discriminating self from externally altered actions for both temporal and spatial alterations. Second, psychosis patients' metacognition of SoA was impaired, and their confidence ratings did not track the accuracy of their SoA judgments. Finally, using a data driven approach to classify psychosis patients based on their embodied SoA task performance yielded high classification rates, suggesting that our task may be clinically useful in the detection and monitoring of psychotic states.

Psychosis patients showed a considerable deficit in their ability to judge whether the movement of the $\mathrm{VH}$ was identical to their actual movement or altered. For both temporal and spatial alternations their sensitivity to sensorimotor conflicts was significantly reduced compared to the control group, and they tended to erroneously 
attribute actions to themselves (i.e., an over-attribution of SoA). This impairment is in line with previous reports of reduced abilities to predict the outcomes of one's actions in schizophrenia (Lindner et al., 2005; Synofzik et al., 2010; Voss et al., 2010). It has been previously suggested that abnormal temporal predictions and processing may underlie these SoA deficits (Graham-Schmidt et al., 2016; Koreki et al., 2015; Waters \& Jablensky, 2009; Whitford et al., 2012). However the current findings indicate that embodied sensorimotor predictions in the spatial domain are also compromised (Franck et al., 2001; Synofzik et al., 2010). In line with accounts highlighting disturbances of embodiment and the Self in psychosis (Hur et al., 2014; Sass \& Parnas, 2003), our findings provide support for an impairment in the processing of the Self that extends across different perceptual dimensions.

In addition to SoA performance, we also investigated participants' metacognition of SoA. While psychosis patients had comparable overall levels of confidence in their SoA judgments, this contrasted strongly with their low level of accuracy. Converging evidence from the mixed model analysis and gamma-ranked correlations indicate that while the control participants' confidence tracked their SoA accuracy, this metacognition of SoA was absent in psychosis patients. Deficits of metacognitive capacities are well documented across the schizophrenia spectrum and has been related to poorer outcomes (Dietrichkeit et al., 2020; Hasson-Ohayon et al., 2018; Koren et al., 2006; Lysaker et al., 2011). However, recent work on perceptual metacognition indicates that when task difficulty is stringently controlled, metacognitive deficits in schizophrenia are small or even absent (Faivre, Roger, et al., 2020; Powers et al., 2017; Rouy et al., 2021). The current study examining embodied SoA in patients, found an extensive 
deficit in SoA discrimination, combined with high confidence in their judgments pointing to a considerable deficit in metacognition for SoA (although, one must take into account that first order performance was not equated here, which may account for some of the differences between the groups, see Rouy et al., 2021). This suggests that in contrast to low-level perceptual metacognitive capacities which may be preserved, metacognition of SoA involving the integration of sensorimotor signals and higher-order constructs such as beliefs and intentions is severely impaired. This deficit is of clinical interest as the lack of SoA abilities compounded by their unawareness of this deficit, may relate to patients' lack of insight into clinical symptoms such as hallucinations and delusions (American Psychiatric Association, 2013; Koren et al., 2006). Interestingly, an exploratory analysis revealed a strong and significant correlation between GK metacognitive measure and positive symptoms in the psychosis patient group $(r=-0.47$, $p<0.01$, see Table 2). Thus, of all experimental measures, metacognitive ability was most strongly related to psychosis symptoms, yet further research is needed to robustly examine this relation between metacognition of SoA and psychosis symptoms.

Psychosis patients showed a higher tendency to erroneously attribute actions to themselves, and while this self-attribution bias has been shown in previous studies with psychosis patients (e.g., Daprati et al., 1997; Franck et al., 2001; Hauser et al., 2011), it stands in stark contrast to clinical symptoms of psychosis such as passivity symptoms in which reduced agency is experienced (Synofzik et al., 2010). It has been suggested that the over-attribution bias may originate from more explicit top-down processes that take into account intentions, beliefs and contextual information in forming judgments of agency that compensate for the lack of sensorimotor signals that typically form the basis 
for SoA (Synofzik et al., 2008, 2009). Indeed, reduced precision of sensorimotor predictive models may lead to overweighting top-down priors, causing an overattribution of SoA to the Self (Corlett et al., 2019; Leptourgos \& Corlett, 2020). Our current finding of high subjective ratings of confidence despite low accuracy of SoA performance (i.e., impaired metacognition), support this hypothesis that top-down explicit processes (i.e., 'I moved and saw a movement so it is likely me') may receive higher weightings despite impairments in sensorimotor prediction function in psychosis.

The relation between clinical symptoms and SoA metrics revealed several interesting findings. Contrary to our pre-registered hypothesis, positive symptoms were not significantly correlated with SoA sensitivity $(r=-0.16, p=0.41$, see Table 2$)$ nor were SPQ-B perceptual deficits $(r=-0.32, p=0.08$, see Table 2$)$. An exploratory analysis revealed a correlation between the SPQ-B disorganization scale and bias $(r=0.48, p<$ 0.01 , see Table 2). Indeed, previous studies have shown inconsistent correlations between prodromal symptoms (Asai et al., 2008; Krugwasser et al., 2019; Stern et al., 2020), psychosis symptoms (Graham-Schmidt et al., 2018; Hauser et al., 2011; Hur et al., 2014) and SoA measures. It should be noted that the current study's sample size had low statistical power to detect such correlations.

Finally, we tested whether our embodied SoA paradigm might have clinical utility for identification and monitoring of psychosis. Using a classifier based on individual SoA performance, we were able to classify psychosis patients and controls with high levels of accuracy ( $\sim 90 \%)$. Critically, this finding was robust when using only a small subset of trials or participants. This indicates that the differences in the tuning curve for the Self (i.e., the shape of the SoA slopes) is a strong predictor of psychosis across subjects. 
This is in line with accounts of an expanded sensorimotor temporal or spatial integration windows in psychosis, which may induce a wider "tuning curve" for the Self (Haggard et al., 2003; Synofzik et al., 2009; Voss et al., 2010). At the practical level, such computerized measurements could augment current in-person diagnosis of psychotic states by providing a telehealth option for online diagnosis and monitoring. Future studies employing multiple measurements could assess the relation of SoA to patients' clinical states over the course of hospitalization and recovery.

The current study suffers from several limitations. First, the psychosis cohort was not very large and was diverse in their psychiatric diagnosis (see Table 1). However, we suggest that the robustness of our SoA findings, despite this heterogeneity in the patients group indicates that SoA and metacognitive deficits are a major feature of the psychotic state. Second, as we aimed to test SoA across different levels of sensorimotor ambiguity, our data was not aimed to stringently control for task difficulty and this limited our ability to employ novel metacognitive measures (Faivre, Vuillaume, et al., 2020; Fleming \& Lau, 2014). Future work on metacognition of SoA should control first order performance more stringently. Finally, the control and psychosis groups were not matched for age, however no relations between age and any of the SoA or confidence measures were found (see supplemental Table S2).

In summary, employing an embodied virtual reality paradigm, we showed that psychosis patients are not only significantly impaired in their ability to discriminate their actions, but also show a substantial lack of awareness of this impairment. These results suggest deficits across multiple systems underlying SoA, including both low precision sensorimotor prediction mechanisms causing reduced sensitivity to deviations, as well 
as overreliance on top-down priors causing high confidence in erroneous judgments of agency. Importantly, patients' insight to their difficulties in the demarcation of the Self may provide a foothold for understanding and treating Self disorders in psychosis.

\section{Author Contribution}

Conceptualization, R.S. and E.V.H.; Methodology, R.S. and A.R.K.; Formal analysis, A.R.K., Y.S., N.F. and R.S.; Data curation, A.R.K. and R.S.; Data collection, A.R.K. and E.V.H; Writing - original draft preparation, A.R.K., Y.S., and R.S.; Writing - review and editing, A.R.K., Y.S., N.F. and R.S.; Analysis code, A.R.K. and Y.S.; Visualization, A.R.K. and Y.S.; project administration, A.R.K.; funding acquisition, R.S.; All authors have read and agreed to the published version of the manuscript.

\section{Acknowledgments}

We would like to thank Viktoria Vul for her help in collecting the data and Yair Zvilichovsky for developing the software used to run the experiment. We would also like to thank the patients for taking part in the experiment.

\section{Funding}

This study was supported by Israeli Science Foundation Grant (\#1169/17) and a National Institute for Psychobiology in Israel grant to R.S.

\section{Conflicts of Interest}

The authors declare no conflict of interest. 


\section{$\underline{\text { References }}$}

Allen, M., \& TSakiris, M. (2018). The body as first prior: Interoceptive predictive processing and the primacy. The Interoceptive Mind: From Homeostasis to Awareness, 27.

American Psychiatric Association. (2013). DSM 5. American Psychiatric Association.

Asai, T., Sugimori, E., \& Tanno, Y. (2008). Schizotypal personality traits and prediction of one's own movements in motor control: What causes an abnormal sense of agency? Consciousness and Cognition, 17(4), 1131-1142.

https://doi.org/10.1016/j.concog.2008.04.004

Barr, D. J., Levy, R., Scheepers, C., \& Tily, H. J. (2013). Random effects structure for confirmatory hypothesis testing: Keep it maximal. Journal of Memory and Language, 68(3), 255-278. https://doi.org/10.1016/j.jml.2012.11.001

Bates, D., Mächler, M., Bolker, B., \& Walker, S. (2014). Fitting Linear Mixed-Effects Models using Ime4. ArXiv:1406.5823 [Stat]. http://arxiv.org/abs/1406.5823

Bays, P. M., Flanagan, J. R., \& Wolpert, D. M. (2006). Attenuation of self-generated tactile sensations is predictive, not postdictive. PLoS Biology, 4(2), e28.

Bernasconi, F., Blondiaux, E., Potheegadoo, J., Stripeikyte, G., Pagonabarraga, J., Bejr-Kasem, H., Bassolino, M., Akselrod, M., Martinez-Horta, S., Sampedro, F., Hara, M., Horvath, J., Franza, M., Konik, S., Bereau, M., Ghika, J.-A., Burkhard, P. R., Ville, D. V. D., Faivre, N., ... Blanke, O. (2021). Robot-induced hallucinations in Parkinson's disease depend on altered sensorimotor processing in fronto-temporal network. Science Translational Medicine, 13(591). https://doi.org/10.1126/scitransImed.abc8362 
Blakemore, S.-J., Wolpert, D. M., \& Frith, C. D. (1998). Central cancellation of selfproduced tickle sensation. Nature Neuroscience, 1(7), 635-640.

Blanke, O., Pozeg, P., Hara, M., Heydrich, L., Serino, A., Yamamoto, A., Higuchi, T., Salomon, R., Seeck, M., \& Landis, T. (2014). Neurological and robot-controlled induction of an apparition. Current Biology, 24(22), 2681-2686.

Chambon, V., Filevich, E., \& Haggard, P. (2014). What is the Human Sense of Agency, and is it Metacognitive? In S. M. Fleming \& C. D. Frith (Eds.), The Cognitive Neuroscience of Metacognition (pp. 321-342). Springer Berlin Heidelberg. https://doi.org/10.1007/978-3-642-45190-4_14

Constant, M., Salomon, R., \& Filevich, E. (2021). Judgments of agency are affected by sensory noise without recruiting metacognitive processing. BioRxiv, 2021.04.28.441761. https://doi.org/10.1101/2021.04.28.441761

Corlett, P. R., Horga, G., Fletcher, P. C., Alderson-Day, B., Schmack, K., \& Powers, A. R. (2019). Hallucinations and Strong Priors. Trends in Cognitive Sciences, 23(2), 114-127. https://doi.org/10.1016/j.tics.2018.12.001

Daprati, E., Franck, N., Georgieff, N., Proust, J., Pacherie, E., Dalery, J., \& Jeannerod, M. (1997). Looking for the agent: An investigation into consciousness of action and self-consciousness in schizophrenic patients. Cognition, 65(1), 71-86.

David, N., Newen, A., \& Vogeley, K. (2008). The "sense of agency" and its underlying cognitive and neural mechanisms. Consciousness and Cognition, 17(2), 523534. 
Dietrichkeit, M., Grzella, K., Nagel, M., \& Moritz, S. (2020). Using virtual reality to explore differences in memory biases and cognitive insight in people with psychosis and healthy controls. Psychiatry Research, 285, 112787.

Faivre, N., Roger, M., Pereira, M., de Gardelle, V., Vergnaud, J., Passerieux, C., \& Roux, P. (2020). Confidence in visual motion discrimination is preserved in individuals with schizophrenia. Journal of Psychiatry \& Neuroscience: JPN, 45(6), $200022-200022$.

Faivre, N., Vuillaume, L., Bernasconi, F., Salomon, R., Blanke, O., \& Cleeremans, A. (2020). Sensorimotor conflicts alter metacognitive and action monitoring. Cortex, 124, 224-234.

Fleming, S. M., \& Lau, H. C. (2014). How to measure metacognition. Frontiers in Human Neuroscience, 8. https://doi.org/10.3389/fnhum.2014.00443

Fletcher, P. C., \& Frith, C. D. (2008). Perceiving is believing: A Bayesian approach to explaining the positive symptoms of schizophrenia. Nature Reviews Neuroscience, 10(1), 48-58.

Ford, J. M., Palzes, V. A., Roach, B. J., \& Mathalon, D. H. (2013). Did I do that? Abnormal predictive processes in schizophrenia when button pressing to deliver a tone. Schizophrenia Bulletin, sbt072.

Fourneret, P., Paillard, J., Lamarre, Y., Cole, J., \& Jeannerod, M. (2002). Lack of conscious recognition of one's own actions in a haptically deafferented patient. NeuroReport, 13(4), 541-547. 
Franck, N., Farrer, C., Georgieff, N., Marie-Cardine, M., Daléry, J., d'Amato, T., \& Jeannerod, M. (2001). Defective recognition of one's own actions in patients with schizophrenia. American Journal of Psychiatry, 158(3), 454-459.

Frith, C. D., \& Done, D. J. (1989). Experiences of alien control in schizophrenia reflect a disorder in the central monitoring of action. Psychological Medicine, 19(2), 359363.

Gallagher, S. (2000). Philosophical conceptions of the self: Implications for cognitive science. Trends in Cognitive Sciences, 4(1), 14-21.

Gallagher, S. (2012). Multiple aspects in the sense of agency. New ldeas in Psychology, 30(1), 15-31. https://doi.org/10.1016/j.newideapsych.2010.03.003

Goodman, L. A., \& Kruskal, W. H. (1979). Measures of Association for Cross Classifications. In L. A. Goodman \& W. H. Kruskal (Eds.), Measures of Association for Cross Classifications (pp. 2-34). Springer. https://doi.org/10.1007/978-1-4612-9995-0_1

Graham-Schmidt, K. T., Martin-Iverson, M. T., Holmes, N. P., \& Waters, F. A. V. (2016). When one's sense of agency goes wrong: Absent modulation of time perception by voluntary actions and reduction of perceived length of intervals in passivity symptoms in schizophrenia. Consciousness and Cognition, 45, 9-23. https://doi.org/10.1016/j.concog.2016.08.006

Graham-Schmidt, K. T., Martin-Iverson, M. T., \& Waters, F. A. V. (2018). Self- and other-agency in people with passivity (first rank) symptoms in schizophrenia. Schizophrenia Research, 192, 75-81. https://doi.org/10.1016/j.schres.2017.04.024 
Haggard, P. (2017). Sense of agency in the human brain. Nature Reviews Neuroscience, 18(4), 196-207. https://doi.org/10.1038/nrn.2017.14 Haggard, P., Martin, F., Taylor-Clarke, M., Jeannerod, M., \& Franck, N. (2003). Awareness of action in schizophrenia. NeuroReport, 14(7), 1081-1085. Hasson-Ohayon, I., Goldzweig, G., Lavi-Rotenberg, A., Luther, L., \& Lysaker, P. H. (2018). The centrality of cognitive symptoms and metacognition within the interacting network of symptoms, neurocognition, social cognition and metacognition in schizophrenia. Schizophrenia Research, 202, 260-266.

Hauser, M., Knoblich, G., Repp, B. H., Lautenschlager, M., Gallinat, J., Heinz, A., \& Voss, M. (2011). Altered sense of agency in schizophrenia and the putative psychotic prodrome. Psychiatry Research, 186(2), 170-176. https://doi.org/10.1016/j.psychres.2010.08.003

Hughes, G., Desantis, A., \& Waszak, F. (2013). Mechanisms of intentional binding and sensory attenuation: The role of temporal prediction, temporal control, identity prediction, and motor prediction. Psychological Bulletin, 139(1), 133.

Hughes, G., \& Waszak, F. (2011). ERP correlates of action effect prediction and visual sensory attenuation in voluntary action. Neurolmage, 56(3), 1632-1640. https://doi.org/10.1016/j.neuroimage.2011.02.057

Hur, J.-W., Kwon, J. S., Lee, T. Y., \& Park, S. (2014). The crisis of minimal selfawareness in schizophrenia: A meta-analytic review. Schizophrenia Research, 152(1), 58-64.

Kannape, O., Schwabe, L., Tadi, T., \& Blanke, O. (2010). The limits of agency in walking humans. Neuropsychologia, 48(6), 1628-1636. 
Kass, R. E., \& Raftery, A. E. (1995). Bayes Factors. Journal of the American Statistical Association, 90(430), 773-795.

https://doi.org/10.1080/01621459.1995.10476572

Kay, S., Fiszbein, A., \& Opler, L. (1987). The positive and negative syndrome scale (PANSS) for schizophrenia. Schizophrenia Bulletin, 13(2), 261.

Kilteni, K., \& Ehrsson, H. H. (2017). Body ownership determines the attenuation of selfgenerated tactile sensations. Proceedings of the National Academy of Sciences, 114(31), 8426-8431. https://doi.org/10.1073/pnas.1703347114

Koreki, A., Maeda, T., Fukushima, H., Umeda, S., Takahata, K., Okimura, T., Funayama, M., Iwashita, S., Mimura, M., \& Kato, M. (2015). Behavioral evidence of delayed prediction signals during agency attribution in patients with schizophrenia. Psychiatry Research, 230(1), 78-83.

https://doi.org/10.1016/j.psychres.2015.08.023

Koren, D., Seidman, L. J., Goldsmith, M., \& Harvey, P. D. (2006). Real-World Cognitive—and Metacognitive_Dysfunction in Schizophrenia: A New Approach for Measuring (and Remediating) More "Right Stuff." Schizophrenia Bulletin, 32(2), 310-326. https://doi.org/10.1093/schbul/sbj035

Krugwasser, A. R., Harel, E. V., \& Salomon, R. (2019). The boundaries of the self: The sense of agency across different sensorimotor aspects. Journal of Vision, 19(4), 14-14. https://doi.org/10.1167/19.4.14

Kuznetsova, A., Brockhoff, P. B., \& Christensen, R. H. B. (2017). ImerTest Package: Tests in Linear Mixed Effects Models. Journal of Statistical Software, 82(1), 126. https://doi.org/10.18637/jss.v082.i13 
Leptourgos, P., \& Corlett, P. (2020). Embodied predictions, agency, and psychosis.

Front. Big Data 3: 27. Doi: 10.3389/Fdata.

Lindner, A., Thier, P., Kircher, T. T. J., Haarmeier, T., \& Leube, D. T. (2005). Disorders of Agency in Schizophrenia Correlate with an Inability to Compensate for the Sensory Consequences of Actions. Current Biology, 15(12), 1119-1124. https://doi.org/10.1016/j.cub.2005.05.049

Lysaker, P. H., Carcione, A., Dimaggio, G., Johannesen, J. K., Nicolò, G., Procacci, M., \& Semerari, A. (2005). Metacognition amidst narratives of self and illness in schizophrenia: Associations with neurocognition, symptoms, insight and quality of life. Acta Psychiatrica Scandinavica, 112(1), 64-71. https://doi.org/10.1111/j.1600-0447.2005.00514.x

Lysaker, P. H., \& Dimaggio, G. (2014). Metacognitive Capacities for Reflection in Schizophrenia: Implications for Developing Treatments. Schizophrenia Bulletin, 40(3), 487-491. https://doi.org/10.1093/schbul/sbu038

Lysaker, P. H., Olesek, K. L., Warman, D. M., Martin, J. M., Salzman, A. K., Nicolò, G., Salvatore, G., \& Dimaggio, G. (2011). Metacognition in schizophrenia: Correlates and stability of deficits in theory of mind and self-reflectivity. Psychiatry Research, 190(1), 18-22. https://doi.org/10.1016/j.psychres.2010.07.016

Macmillan, N. A. (2002). Signal detection theory. Stevens' Handbook of Experimental Psychology, 4, 43-90.

Maeda, T., Kato, M., Muramatsu, T., Iwashita, S., Mimura, M., \& Kashima, H. (2012). Aberrant sense of agency in patients with schizophrenia: Forward and backward 
over-attribution of temporal causality during intentional action. Psychiatry Research, 198(1), 1-6.

MATLAB. (2019). MATLAB 9.6.0.(R2019a). The MathWorks Inc.

Moore, J. W., Wegner, D. M., \& Haggard, P. (2009). Modulating the sense of agency with external cues. Consciousness and Cognition, 18(4), 1056-1064.

Palmer, C. E., Davare, M., \& Kilner, J. M. (2016). Physiological and Perceptual Sensory Attenuation Have Different Underlying Neurophysiological Correlates. Journal of Neuroscience, 36(42), 10803-10812. https://doi.org/10.1523/JNEUROSCI.169416.2016

Powers, A. R., Mathys, C., \& Corlett, P. R. (2017). Pavlovian conditioning-induced hallucinations result from overweighting of perceptual priors. Science, 357(6351), 596-600. https://doi.org/10.1126/science.aan3458

R Core Team. (2019). R: A Language and Environment for Statistical Computing. $\mathrm{R}$ Foundation for Statistical Computing. https://www.R-project.org/

Raballo, A., Sæbye, D., \& Parnas, J. (2011). Looking at the Schizophrenia Spectrum Through the Prism of Self-disorders: An Empirical Study. Schizophrenia Bulletin, 37(2), 344-351. https://doi.org/10.1093/schbul/sbp056

Raine, A., \& Benishay, D. (1995). The SPQ-B: A Brief Screening Instrument for Schizotypal Personality Disorder. Journal of Personality Disorders; New York, 9(4), 346-355. http://dx.doi.org/10.1521/pedi.1995.9.4.346

Rouy, M., Saliou, P., Nalborczyk, L., Pereira, M., Roux, P., \& Faivre, N. (2021). Systematic review and meta-analysis of metacognitive abilities in individuals with 
schizophrenia spectrum disorders. Neuroscience \& Biobehavioral Reviews, 126, 329-337. https://doi.org/10.1016/j.neubiorev.2021.03.017

Salomon, R., Lim, M., Pfeiffer, C., Gassert, R., \& Blanke, O. (2013). Full body illusion is associated with widespread skin temperature reduction. Frontiers in Behavioral Neuroscience, 7.

Salomon, R., Progin, P., Griffa, A., Rognini, G., Do, K. Q., Conus, P., Marchesotti, S., Bernasconi, F., Hagmann, P., \& Serino, A. (2020). Sensorimotor Induction of Auditory Misattribution in Early Psychosis. Schizophrenia Bulletin.

Sass, L. A., \& Parnas, J. (2003). Schizophrenia, Consciousness, and the Self. Schizophrenia Bulletin, 29(3), 427-444. https://doi.org/10.1093/oxfordjournals.schbul.a007017

Schneider, K. (1959). Clinical psychopathology. Grune \& Stratton.

Schwarz, G. (1978). Estimating the dimension of a model. The Annals of Statistics, 6(2), 461-464.

Serino, A., Pozeg, P., Bernasconi, F., Solcà, M., Hara, M., Progin, P., Stripeikyte, G., Dhanis, H., Salomon, R., \& Bleuler, H. (2020). Thought consciousness and source monitoring depend on robotically-controlled sensorimotor conflicts and illusory states. IScience, 101955.

Shergill, S. S., Samson, G., Bays, P. M., Frith, C. D., \& Wolpert, D. M. (2005). Evidence for Sensory Prediction Deficits in Schizophrenia. Am J Psychiatry, 162(12), 2384-2386. https://doi.org/10.1176/appi.ajp.162.12.2384

Shergill, S. S., Samson, G., Bays, P. M., Frith, C. D., \& Wolpert, D. M. (2014). Evidence for sensory prediction deficits in schizophrenia. American Journal of Psychiatry. 
Shergill, S. S., White, T. P., Joyce, D. W., Bays, P. M., Wolpert, D. M., \& Frith, C. D. (2012). Modulation of somatosensory processing by action. Neuroimage.

Shimada, S., Fukuda, K., \& Hiraki, K. (2009). Rubber Hand Illusion under Delayed Visual Feedback. PLOS ONE, 4(7), e6185. https://doi.org/10.1371/journal.pone.0006185

Stern, Y., Koren, D., Moebus, R., Panishev, G., \& Salomon, R. (2020). Assessing the Relationship between Sense of Agency, the Bodily-Self and Stress: Four VirtualReality Experiments in Healthy Individuals. Journal of Clinical Medicine, 9(9), 2931.

Sterzer, P., Adams, R. A., Fletcher, P., Frith, C., Lawrie, S. M., Muckli, L., Petrovic, P., Uhlhaas, P., Voss, M., \& Corlett, P. R. (2018). The Predictive Coding Account of Psychosis. Biological Psychiatry, 84(9), 634-643.

https://doi.org/10.1016/j.biopsych.2018.05.015

Stripeikyte, G., Pereira, M., Rognini, G., Potheegadoo, J., Blanke, O., \& Faivre, N. (2020). Increased functional connectivity of the intraparietal sulcus underlies the attenuation of numerosity estimations for self-generated words. BioRxiv, 2020.12.18.423390. https://doi.org/10.1101/2020.12.18.423390

Synofzik, M., Thier, P., Leube, D. T., Schlotterbeck, P., \& Lindner, A. (2010). Misattributions of agency in schizophrenia are based on imprecise predictions about the sensory consequences of one's actions. Brain, 133(1), 262-271.

Synofzik, M., Vosgerau, G., \& Lindner, A. (2009). Me or not me-An optimal integration of agency cues? Consciousness and Cognition, 18(4), 1065-1068. 
Synofzik, M., Vosgerau, G., \& Newen, A. (2008). Beyond the comparator model: A multifactorial two-step account of agency. Consciousness and Cognition, 17(1), 219-239.

Van Elk, M., Salomon, R., Kannape, O., \& Blanke, O. (2014). Suppression of the N1 auditory evoked potential for sounds generated by the upper and lower limbs. Biological Psychology, 102, 108-117.

Voss, M., Moore, J., Hauser, M., Gallinat, J., Heinz, A., \& Haggard, P. (2010). Altered awareness of action in schizophrenia: A specific deficit in predicting action consequences. Brain, 133(10), 3104-3112.

Wagenmakers, E.-J. (2007). A practical solution to the pervasive problems ofp values. Psychonomic Bulletin \& Review, 14(5), 779-804. https://doi.org/10.3758/BF03194105

Waters, F., \& Jablensky, A. (2009). Time discrimination deficits in schizophrenia patients with first-rank (passivity) symptoms. Psychiatry Research, 167(1-2), 1220.

Wen, W., Yamashita, A., \& Asama, H. (2015). The influence of action-outcome delay and arousal on sense of agency and the intentional binding effect. Consciousness and Cognition, 36, 87-95. https://doi.org/10.1016/j.concog.2015.06.004

Whitford, T. J., Ford, J. M., Mathalon, D. H., Kubicki, M., \& Shenton, M. E. (2012). Schizophrenia, myelination, and delayed corollary discharges: A hypothesis. Schizophrenia Bulletin, 38(3), 486-494. 
Wolpert, D. M., Ghahramani, Z., \& Jordan, M. I. (1995). An internal model for sensorimotor integration. Science, 269(5232), 1880. 